\title{
Atención de salud integral del niño
}

DR. GUillermo REPETTO D. *

La atención médica integral es un área del conocimiento destinada a satisfacer la necesidad social de fomentar y mantener la salud, prevenir y aliviar la enfermedad. Este propósito se cumple dentro de un sistema que funciona como un todo integrado en distintos niveles de atención: ambulatoria, de urgencia, de especialidades, cerrada, etc. En la medida en que se eleva la calidad de una de sus partes, todas las restantes se benefician.

El consultorio periférico, sitio en que se efectúa el primer contacto con el paciente es siempre la unidad básica, sea cual sea la organización de salud que se adopte. Ello es especialmente válido en el área materno-infantil. En él se realiza el mayor número de consultas; establece un enlace entre la comunidad y el Hospital y, a la vez, orienta y regula el ingreso a éste. Hace posible la continuidad de la atención, reduce los costos y permite ampliar la cobertura y la capacidad de los servicios. Sólo en él se integran naturalmente los cuidados de salud, eliminando la barrera entre curación y prevención.

La historia ha demostrado que la salud infantil mejora sensiblemente cuando se eleva el nivel de vida, aún cuando se excluya la acción médica $(1,2,3)$; pero ésta es también capaz por sí sola de influenciarla positivamente, y de ello hay sobradas demostraciones $(4,5,6)$. Pero, para que esto ocurra, es necesario que la primera línea de contacto, responsable de la atención del núcleo familiar, realice una medicina integral otorgando prioridad a las acciones de protección y fomento. En el área de atención pediátrica esto es sinónimo de "supervisión del crecimiento y desarrollo, prevención, observación y tratamiento oportuno de sus desviaciones". Un programa de tal naturaleza,

\footnotetext{
* Departamento de Pediatría. Facultad de Medicina. Sede Santlago Norte. Hospital Roberto del Río.
}

realizado en un local adecuado y accesible, sólo adquiere racionalidad cuando tiene presente las características esenciales de dicho proceso de crecimiento y desarrollo; y por lo tanto, dentro de una política de salud, debe ser: prioritario, integrado a la atención prenatal, de iniciación precoz, continuo y completo $(7,8)$.

En el ejercicio de la atención médica no se observa concordancia entre esa doctrina y su práctica. Se ha dicho que "la atención del Consultorio Externo es uniformemente mala, deshumanizada, impersonal, superficial y frustrante para el que la solicita y para el médico que la dispensa" (9). Aunque se acepte para esta discordancia una causalidad múltiple, el obstáculo principal, progresivo e insalvable, es el crecimiento de la demanda que no aumenta a igual ritmo que los recursos, problema que también preocupa a los países desarrollados al punto que "el déficit de personal para el cuidado de la salud es la carencia más importante de fuerza de trabajo en los Estados Unidos" (10). Entre nosotros el problema se agudiza en los grandes núcleos urbanos en donde se generan las mayores cifras de enfermedad y muerte infantil. En la práctica, el médico que labora en un Consultorio Periférico dedica casi todos sug esfuerzos a satisfacer la demanda ocasionada por lo que peyorativamente se suele denominar "patología banal, simple o subpatología", que aumenta día por día. Esta morbilidad responde a medidas terapéuticas simples, a veces sólo sintomáticas y, con frecuencia, es autolimitada en su evolución; pero suele olvidarse que en ellas se ofrece la oportunidad de realizar acciones educativas, de fomento y prevención que sí tienen una influencia favorable en la salud infantil. La restauración de la salud pasa a ser un medio para alcanzar esa finalidad fundamental; en consecuencia, esta consulta debiera ser estimulada y prodigada sin limi- 
taciones y sin obstáculos, teniendo presente las necesidades reales de salud del niño y no sólo las legítimas aspiraciones de la madre que no necesariamente concuerdan.

La solución tradicional de este problema exige aumentar el número de personal calificado necesario para resolver los problemas de salud del país y racionalizar su distribución. Sin embargo, el tiempo requerido para la formación de un médico o de una enfermera será siempre prolongado, y la persecución de una meta ideal no puede invalidar la necesidad de buscar nuevos modelos asistenciales más acordes con la realidad actual.

Son muchas las iniciativas que a este respecto se han sugerido; casi todas constituyen soluciones parciales, aunque no excluyentes. Se ha propuesto, por ejemplo, crear un médico de "choque" o de "vanguardia" que atienda la patología frecuente y derive a los médicos de "retaguardia" los casos de diagnóstico dudoso o los graves. Aunque con ello puede aumentarse el rendimiento y reducir o eliminar los rechazos, el énfasis, una vez más, está en lo curativo con olvido de lo verdaderamente importante que es la prevención y el fomento de la salud, única medida capaz de reducir la demanda por enfermedad.

El concepto de alto riesgo biológico $(11,12$, 13) también ofrece una vía no explorada. Pretende identificar los subgrupos de población más afectos a daños susceptibles a la acción médica y, así, aumentar el rendimiento haciendo mejor uso de recursos siempre limitados. En esta misma línea de pensamientos, la Oficina Sanitaria Panamericana ha introducido el concepto de "cuidado progresivo", de acuerdo al cual se organizan los servicios según las necesidades del paciente (14). Por ahora, su traducción práctica ha sido la creación de Unidades de Cuidados Intensivo, pero "los servicios ambulatorios constituyen la base fundamental más sólida donde debe afirmarse la pirámide de la atención progresiva" (15), y en ello poco se ha avanzado a la fecha.

Por desgracia, el grupo expuesto a alto riesgo biológico en Chile es demasiado numeroso para tratar de encontrar una solución sólo por las vías enumeradas. Basta recordar que el $21 \%$, esto es, uno de cada cinco chilenos vive en estado de "extrema pobreza" (16), situación que de facto los ubica en el grupo de elevado riesgo.

Si se analizan, una vez más, los problemas de salud del niño que destacan por su frecuencia y gravedad se aprecia que casi todos son susceptibles a medidas preventivas y terapéuticas sencillas que incluyan un componente educativo continuado, sistematizado y al alcance de la madre. Muchas pueden ser efectuadas con eficiencia por profesionales de colaboración médica, auxiliares o voluntarias debidamente capacitadas, siempre que el médico conserve activamente el liderazgo y la responsabilidad, establezca la necesaria supervisión y una fácil y pronta comunicación entre los distintos niveles, adaptados, naturalmente, según se trate de un consultorio metropolitano o rural. Con ello no se pretende crear una barrera o un filtro entre el paciente y el médico, sino, más bien, lograr que éste trabaje "con" y "a través" de personal menos capacitado en la procura de un objetivo común, multiplicando, de este modo, su campo de acción; y ello, dentro de una estructura que establezca niveles de complejidad técnica progresiva y que reemplace la "atención médica por médicos" por una "atención para la salud por el equipo de salud".

Una decisión de la naturaleza sugerida obliga previamente a un análisis detenido de los aspectos éticos envueltos, para definir aquellas acciones exclusivas del médico que son, por lo tanto, indelegables; así como para no entorpecer la entrega de servicios que pueden ser ejecutados con eficiencia por otros integrantes del equipo de salud y que son, quizás, los que con mayor urgencia requieren nuestras comunidades.

En resumen, el modelo asistencial que se propone explorar a través de estas líneas consistiría en:

1. Ampliar la base del equipo multidisciplinario de salud delimitando claramente niveles de atención de menor a mayor complejidad;

2. Asignar a personal no-profesional responsabilidad en acciones de salud definidas, en una primera línea de contacto y con un carácter longitudinal e integral.

Un programa de esta naturaleza haría verdaderamente accesible la medicina integral requerida por los niños de nuestros grupos sociales desvalidos; permitiría ampliar la utilización de los consultorios periféricos instalados en una fórmula que no contradice la actual política económica y liberaría recursos de mayor nivel técnico para desarrollar tareas en las cuales son irreemplazables.

El camino ya ha sido emprendido por nacio nes de mayor desarrollo que el nuestro. (17-18-19. 20-21-22-23).

\section{REFERENCLAS}

1.-Mc Keown T. $y$ Record, R. G. Reasons for the decline of mortality in England and Wales during the nineteenth century. Population Studies 16: 94, 1962 (Nov.).

2.-Mc Keown T. y Brown, R. E. Medical evidence related to English Population changes in the eighteenth century. Population studies 9: 119, 1955. 
3.- Joseph M. and Keith, R. A new look at child health. Pitman Medical Publ. Co., London, 1966.

4.-Repetto, G. y Soza, G. Mortalidad en la niñez debida a enfermedades prevenibles mediante vacunas. Rev. Médica Chile, 102: 550, 1974.

5.-Behm, H. Mortalidad Infantil y nivel de vida. Univ. de Chile, Santiago, 1962.

6.-Majluf, S. Romero, M. I., Ubilla G., Guerrero, M. y Palacios, G. Nivel de Salud y atención pediátrica preventiva. Escuela de Ingeniería. Departamento de Ingeniería. Departamento de Ingenieria de Sistemas. Universidad Católica de Chile. Enero, 1975.

7.-Adriasola, G. y Plaza, S. Curso diversificado de higiene materna e infantil. Escuela de Salubridad. Univ. de Chile, Santiago, 1961.

8.-González, S. El proceso de crecimiento y desarrollo en la atención infantil. En: Meneghello, J. Pediatría. Intermédica, Buenos Aires, 1972.

9.-Colegio Médico de Chile. Política de salud propiciada por el Colegio Médico de Chile, Rev. Med. Chile 102: 1974.

10.- Carnegie Commission on Higher Education. Higher education on the Nation's health. McGraw-Hill Book Co., New York, 1970.

11.- Montoya, C. Aplicaciones del concepto de riesgo en salud materno-infantil. Bol. Of. Sanit. Panam. 77: 93, 1974.

12.-Butler, N. R. and Alberman E. Perinatal problems. The second report of the 1958 British perinatal mortality survey. Edinburgh, E. and S. Livingstone Ltd., 1968.

13.-Sheridan, $M$. Infants at risk of handicapping conditions. Mont, Bull. Min. Heath, 21: 238, 1962.
14.- Dávila, C. Unidades de Cuidado Intensivo en América Latina. Bol. Of. Sanit. Panam. 68: 50, 1970.

15.-Dávila, C. Propósitos y objetivos del seminario sobre servicios de atención ambulatoria. Bello Horizonte, Brasil, Of. Sant. Panam. Agosto, 1973. (mimeografiado).

16.-Molina S. y Kast, Miguel. Odeplan Instituto de Economía, Úniversidad Católica de Chile. Mapa de la extrema pobreza en Chile, Documento Reportaje. El Mercurio, Santiago, Jueves 10 de Octubre, 1974 .

17.- Silver, $H$. $K$. The use of new types of allied professionals in providing care for children. Am. J. Dis. Child 116: 486, 1968.

18.- Schiff, D. Fraser Ch. and Walters $H$. The pediatric nurse practitioner in the office of pediatricians in private practice Pediatrics 44: 62, 1969.

19.- Siegel, $B$. Organization of the primary care team Pediatrics Clin. North Am. 21: 341, 1974.

20.- Yodfat. Y. A new method of teamwork in family medicine in Israel with the participation of nurses as physician's assistants. Am. J. Public. Health 62: $953,1972$.

21.-Ostegard, $D$. The potential for paramedical person nel in family planning; an analysis based on the department of Health, Education and Welfore five year plan for family planning servics. Am. J. Public Health 64: 27-31, 1974.

22.-Morley David. Paediatric Priorities in the developing world. Postgraduate Paediatrics Series, Buttherworths, London, 1973.

23.- Medicina simplificada de Venezuela. Ofic. San. Pan. Gaceta Vol. 5, No 4, 1973. 